Document downloaded from:

http://hdl.handle.net/10251/60324

This paper must be cited as:

Avsarkisov, V.; Oberlack, M.; Hoyas Calvo, S. (2014). New scaling laws for turbulent Poiseuille flow with wall transpiration. Journal of Fluid Mechanics. 746:99-122. doi:10.1017/jfm.2014.98.

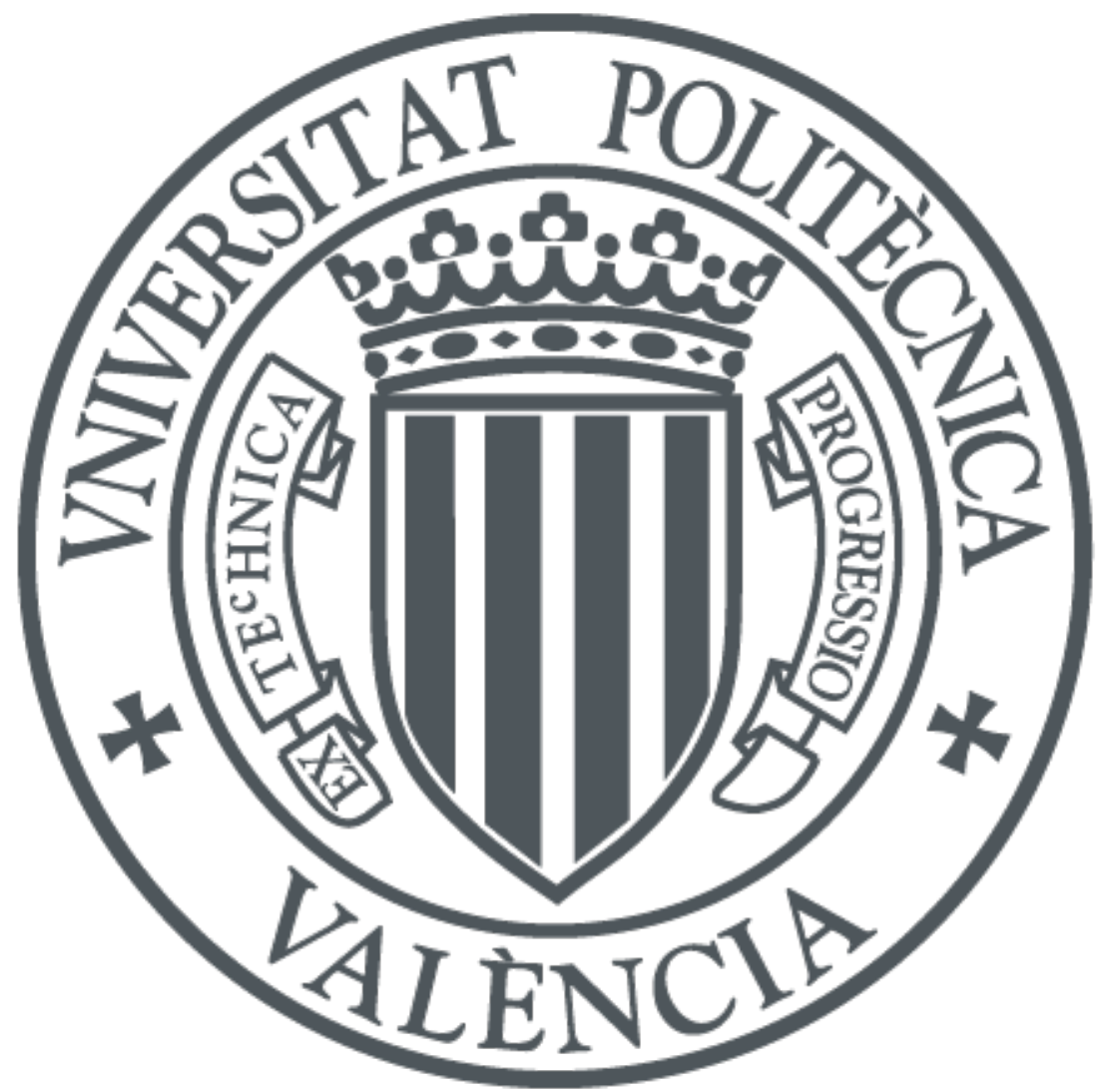

The final publication is available at

http://dx.doi.org/10.1017/jfm.2014.98

Copyright Cambridge University Press (CUP): STM Journals

Additional Information 


\title{
New Scaling Laws for Turbulent Poiseuille Flow with Wall Transpiration
}

\author{
V. A VSARKIS O V ${ }^{1} \dagger$, M. OB ERLA C K $\mathbf{K}^{1,2,3}$, \\ S. HOY A S ${ }^{4}$ AND G. K H U J D Z E \\ ${ }^{1}$ Chair of Fluid Dynamics, TU Darmstadt, Otto-Berndt-Strasse 2, 64287 Darmstadt, Germany \\ ${ }^{2}$ Center of Smart Interfaces, TU Darmstadt, Alarich-Weiss-Str. 10, 64287 Darmstadt, Germany \\ ${ }^{3}$ GS Computational Engineering, TU Darmstadt, Dolivostr. 15, 64293 Darmstadt, Germany \\ ${ }^{4}$ CMT Motores Térmicos, Univ. Politècnica de València, València, Spain
}

(Received ?; revised ?; accepted ?. - To be entered by editorial office)

A fully developed, turbulent Poiseuille flow with wall transpiration, i.e. uniform blowing and suction on the lower and upper walls correspondingly, is investigated by both direct numerical simulation (DNS) of the three-dimensional, incompressible Navier-Stokes equations and Lie symmetry analysis. The latter is used to find symmetry transformations and in turn to derive invariant solutions of the set of two- and multi-point correlation equations. We show that the transpiration velocity is a symmetry breaking which implies a logarithmic scaling law in the core of the channel. DNS validates this result of Lie symmetry analysis and hence aids establishing a new logarithmic law of deficit-type.

The region of validity of the new logarithmic law is very different from the usual near-wall log-law and the slope constant in the core region differs from the von Kármán constant and is equal to 0.3. Further, extended forms of the linear viscous sublayer law and the near-wall log-law are also derived, which, as a particular case, include these laws for the classical non-transpirating case. The viscous sublayer at the suction side has an asymptotic suction profile. The thickness of the sublayer increase at high Reynolds and transpiration numbers. For the near-wall log-law we see an indication that it appears at the moderate transpiration rates $\left(0.05<v_{0} / u_{\tau}<0.1\right)$ and only at the blowing wall. Finally, from the DNS data we establish a relation between the friction velocity $u_{\tau}$ and the transpiration $v_{0}$ which turns out to be linear at moderate transpiration rates.

Key words: Scaling law; Wall transpiration; Lie group analysis; DNS

\section{Introduction}

In this paper we investigate the mean velocity scaling laws for a turbulent Poiseuille flow with uniform wall transpiration as it is shown in figure 1. Wall-bounded turbulent flows with transpiration may not only be a technologically important subject of investigation (Jiménez et al. 2001; Kametani \& Fukagata 2011) but also important for theoretical reasons as we will subsequently show.

In the classical non-transpirating plane turbulent channel flow all statistical quantities are symmetric or antisymmetric with respect to the channel centerline. This includes symmetric distribution of the mean velocity, the normal Reynolds stresses, and point symmetric distributions of viscous and turbulent shear stresses including values of shear stresses at the walls. These results are consistent with zero total shear stress and zero

$†$ Email address for correspondence: avsarkis@fdy.tu-darmstadt.de 


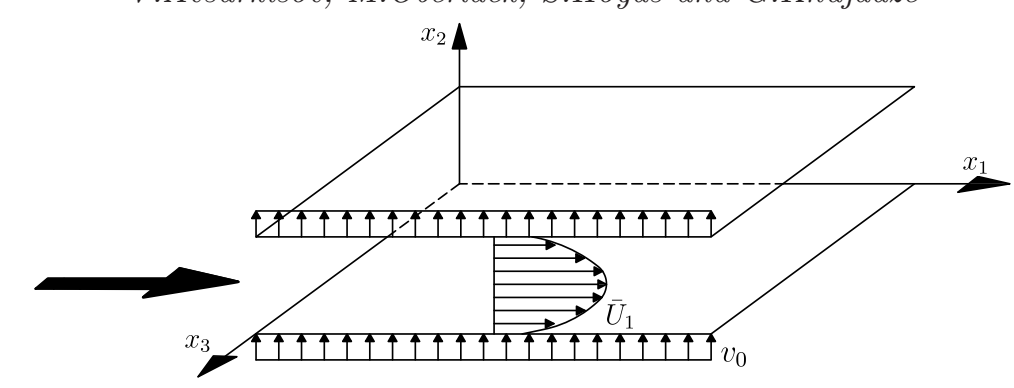

Figure 1. Schematic view of the channel flow with wall transpiration. Fluid is blown through the lower wall and removed from the upper wall with a constant rate.

mean velocity gradient in the center of the channel. In the presence of wall transpiration, all these symmetries are broken. Furthermore, the occurrence of an additional term in the streamwise component of the mean momentum equation modifies the classical universal scaling laws (linear viscous sublayer and law of the wall) for non-transpirating wallbounded flows.

Boundary layer flows with wall transpiration are the most commonly studied wallbounded flow with permeable boundary conditions. First experimental results and a new mean velocity scaling law (so called "bi-logarithmic law") on the subject were obtained in the 1950s (Black \& Sarnecki 1958; Mickley \& Davis 1957) using mixing-length theory. Tennekes (1965) obtained a new form of the law of the wall ("semi-logarithmic") and a velocity defect law assuming the existence of a joint velocity scale $u_{\tau}^{2} / v_{0}$ for the inner and the outer regions. Later Stevenson in his two companion papers (Stevenson 1963a,b) compared his experimental results to the experimental data of Black \& Sarnecki (1958) and Mickley \& Davis (1957). He found generalized forms of the law of the wall and shortly afterwards a velocity defect law for the turbulent boundary layer with suction and blowing at zero pressure gradient. He stated that the slope constant, i.e. the von Kármán constant, and the additive constant $C$ are both independent of the transpiration velocity $v_{0}$. The most recent study on turbulent boundary layer flow with transpiration is a numerical one conducted by Schlatter \& Örlü (2011). They showed that uniform suction considerably changes the mean velocity profile and they found the modified coefficients for the log-law to be $\kappa=0.82$ and $C=9.2$.

In comparison to the other wall-bounded flows with specific, non-standard boundary conditions, turbulent channel i.e. Poiseuille flow with wall transpiration is a relatively new subject of investigation. The only experimental study of this flow of an incompressible fluid known to the authors was conducted by Zhapbasbayev \& Isakhanova (1998). They collected statistics for the mean velocity and turbulent stresses for different Reynolds numbers and a variety of small transpiration velocity numbers in the range $0<v_{0} / u_{\tau}<$ 0.05. Thereafter, they employed the experimental data to evaluate a Reynolds stress transport model developed by Launder and co-authors (see e.g. Hanjalić \& Launder 1972b; Launder et al. 1975).

In the literature only a few DNS studies of the turbulent channel flow with blowing and suction were reported. Sumitani \& Kasagi (1995) studied turbulent channel flow with uniform wall transpiration and heat transfer. The walls were kept isothermal, while their temperatures vary. The Reynolds number and the transpiration rate were held constant at $R e_{\tau}=150$ and $v_{0} / u_{\tau}=0.05$. Various statistical quantities including mean velocity and mean temperature, Reynolds stresses, and turbulent heat fluxes were obtained. Energy budgets and temperature correlations were also calculated. One key overall result they found was that blowing stimulates the near-wall turbulence and creates an excessive 
amount of small scale coherent streamwise vortical structures, while suction suppresses turbulence and at the same time creates large scale near-wall coherent structures. Nikitin \& Pavel'ev (1998) performed DNS computations at $R e_{\tau}=356,681.2$ for $v_{0} / u_{\tau}=0.112$, 0.118 , respectively. They showed that blowing and suction increase the friction coefficient. Apart from this, they investigated the near-wall logarithmic region of the mean velocity profile and found that the slope constant of the log-law at the blowing wall is not constant and increases with the increase of transpiration rate. It is important to mention that they used the local friction velocity at each wall as the velocity scale. Presently we employ an averaged friction velocity from both walls, which is a measure of the pressure gradient. Chung \& Sung (2001) investigated the initial relaxation of a turbulent channel flow after a sudden application of blowing and suction. Chung et al. (2002) analyzed the effects of uniform wall blowing and suction by modulating the near-wall turbulence. They confirmed that suction increases the turbulence anisotropy, while blowing decreases the anisotropy in the near-wall region and enhances the transverse components of the velocity fluctuations $\left(u_{2}\right.$ and $\left.u_{3}\right)$.

A purely analytical study of the turbulent channel flow with wall transpiration was performed by Vigdorovich \& Oberlack (2008). Employing the method of matched asymptotic expansions led them to the construction of the solutions for the near wall regions (both blowing and suction) as well as for the core region of the flow. The results therein did not give any scaling for the mean velocity or the correlation functions but it allowed to describe the relation between the wall shear stress, the Reynolds number, and the transpiration velocity by a function of one variable.

Summarizing all above mentioned studies we conclude that there is no comprehensive investigation of the mean velocity scaling laws of the Poiseuille flow with uniform wall transpiration based on first principles. This difficulty may be traced back to the problem of determining an appropriate velocity scale as there are multiple including $v_{0}, u_{\tau}$ on both walls and $U_{B}$ being the bulk velocity, as well as the proper choice of equations on which to base the analysis.

Presently, the application of Lie symmetry method to the two- and multi-point correlation equations is employed as the fundamental basis to find new mean velocity scaling laws as well as the proper scales on which it is based. The DNS facilitates the evaluation of the analytical results and finally allows us to establish a clear connection between the different velocity scales.

Symmetry analysis of a system of differential equations based on continuous transformation groups, i.e. Lie groups, was introduced by Sophus Lie in the nineteenth century to unify and extend various specialized methods for solving differential equations. The symmetry of a system of differential equations is a transformation that maps any solution to another solution of the system. The advantage of Lie group method, and in turn constructing symmetry transformations, is that they can be found using computational methods. Finding of the symmetry transformations of the two-point correlation (TPC) equations gives profound insight into the flow physics. Once the symmetries are derived, they can be used to achieve reduction or self-similarity in a general sense of the TPC equations. This reduction is always associated with the decrease of the number of independent variables and finally leads to the desired turbulent scaling laws. Presently, the main goal is to find mean velocity scaling laws, but Lie symmetry method is also useful for finding of scaling laws of any higher order statistical quantity.

In a series of papers Oberlack and co-authors (see Oberlack 2000, 2001; Oberlack \& Rosteck 2010; Rosteck \& Oberlack 2011) studied the turbulent channel and other canonical wall-bounded flows using Lie symmetry theory by investigating the infinite series of multi-point correlation (MPC) equations. Thereof they derived a variety of 
classical and new scaling laws. It was shown that they are exact solutions of symmetry invariant type of the infinite dimensional series of MPC equations. They have shown that turbulent scaling laws may be generated from first principle and that most of the classical and new symmetry invariant solutions are based on one or several of the newly discovered statistical symmetry groups (Oberlack \& Rosteck 2010).

In this paper we propose new scaling laws for turbulent Poiseuille flows with wall transpiration including the canonical flow without transpiration as a particular case. Besides, we obtain a new logarithmic scaling law in the center of the channel using Lie symmetry methods. The law is of defect type and covers up to $75 \%$ of the channel depending on the turbulent Reynolds number $R e_{\tau}$ and the transpiration velocity $v_{0}$. In order to validate the new scaling laws and to study the interplay between Reynolds and transpiration number effects, various DNS of the channel flow at $R e_{\tau}=250,480,850$ and a wide range of the transpiration velocity $v_{0}$ are conducted.

Governing equations for the flow with wall transpiration are given in $\S 2$. A new loglaw will be derived in the $\S 3$ and also it will be shown that transpiration generalizes the classical laws for the viscous sublayer and the overlap region. DNS verification of the new scaling law is presented in $\S 4$. Discussion and conclusions are given in $\S 5$.

\section{Basic equations for the turbulent channel flow and DNS}

The analysis presented below is based on the mean friction velocity defined as follows

$$
u_{\tau} \equiv \sqrt{\frac{u_{\tau b}^{2}+u_{\tau s}^{2}}{2}}=\sqrt{\frac{1}{\rho} \frac{\left|\tau_{w b}\right|+\left|\tau_{w s}\right|}{2}}=\sqrt{\frac{h}{\rho}\left|\frac{\partial \bar{P}}{\partial x_{1}}\right|},
$$

which is a measure of the pressure gradient and the local friction velocities are defined as

$$
u_{\tau b}=\sqrt{\nu\left|\frac{\partial \bar{U}_{1}}{\partial x_{2}}\right|_{b}}, \quad u_{\tau s}=\sqrt{\nu\left|\frac{\partial \bar{U}_{1}}{\partial x_{2}}\right|_{s}}
$$

Here, $\bar{U}_{1}$ and $\frac{\partial \bar{P}}{\partial x_{1}}$ are the mean velocity and mean pressure gradient in streamwise direction, $\nu$ is the kinematic viscosity and $h$ is the channel half-width. Here and subsequently subscripts ()$_{b}$ and ()$_{s}$ correspond to variables taken on the blowing and the suction side respectively. For variables at the wall we use the subscript ()$_{w}$ and variables without blowing and suction are denoted by ()$_{0}$. Dimensionless variables in the near-wall scaling will have the superscript plus:

$$
x_{i}^{+}=\frac{x_{i} u_{\tau}}{\nu}, \quad \bar{U}_{i}^{+}=\frac{\bar{U}_{i}}{u_{\tau}}, \quad \overline{u_{i} u_{k}}+=\frac{\overline{u_{i} u_{k}}}{u_{\tau}^{2}}, \quad v_{0}^{+}=\frac{v_{0}}{u_{\tau}}, \quad \tau^{+}=\frac{\tau}{\left(\left|\tau_{w b}\right|+\left|\tau_{w s}\right|\right) / 2} .
$$

Note, that here $u_{\tau}$ is the mean friction velocity, which is a global parameter. Specific differences on each wall and the corresponding differences in scaling will be discussed in the subsections 3.2,3.3 and 3.4. We employ the channel half-width $h$ as a core-region length scaling parameter. The bulk mean velocity is defined as

$$
U_{B}=\frac{1}{2 h} \int_{0}^{2 h} \bar{U}_{1}\left(x_{2}\right) d x_{2} .
$$

It is suitable to start the analysis with the Navier-Stokes equations written in Reynolds averaged form. Throughout this paper we use the following notations. The statistically 
averaged quantities are denoted by an overbar e.g. $\bar{U}_{i}, \bar{P}$ whereas fluctuating quantities are denoted by a lower case letters i.e. $u_{i}$ and $p$. The governing equations for an incompressible turbulent flow, i.e. continuity and mean-momentum equations, are

$$
\begin{gathered}
\frac{\partial \bar{U}_{k}}{\partial x_{k}}=0 \\
\frac{\partial \bar{U}_{i}}{\partial t}+\bar{U}_{k} \frac{\partial \bar{U}_{i}}{\partial x_{k}}=-\frac{1}{\rho} \frac{\partial \bar{P}}{\partial x_{i}}+\nu \frac{\partial^{2} \bar{U}_{i}}{\partial x_{k} \partial x_{k}}-\frac{\partial \overline{u_{i} u_{k}}}{\partial x_{k}}, i=1,2,3,
\end{gathered}
$$

where $\bar{U}_{i}\left(x_{i}, t\right)$ and $\bar{P}\left(x_{i}, t\right)$ are the mean velocity and mean pressure, and $\overline{u_{i} u_{k}}$ is the Reynolds stress tensor. For the incompressible flow investigated, pressure can be normalized with the constant density as follows

$$
\bar{P}^{*}=\frac{\bar{P}}{\rho} .
$$

The asterisk will be omitted throughout the paper. For the present flow we have the following boundary condition (BC) at the wall

$$
\bar{U}_{i}\left(x_{1} ; x_{2}=0,2 h ; x_{3}\right)=\left(0 ; v_{0} ; 0\right)^{T} .
$$

Because of the periodic BC in streamwise direction and general homogeneity in this direction, continuity leads to a constant wall-normal velocity across the channel height, i.e.

$$
\bar{U}_{2}\left(x_{2}\right)=v_{0}
$$

As we employ the BC $U_{i}\left(x_{1} ; x_{2}=0,2 h ; x_{3}\right)=\left(0 ; v_{0} ; 0\right)^{T}$ for the DNS together with $(2.8)$ it implies that all fluctuations vanish at the wall, i.e. $u_{i}=0$. Therefore all Reynolds stresses also vanish at the wall.

With this, we obtain the streamwise component of mean momentum equation for the steady state

$$
v_{0} \frac{d \bar{U}_{1}}{d x_{2}}=-\frac{d \bar{P}}{d x_{1}}-\frac{d \overline{u_{1} u_{2}}}{d x_{2}}+\nu \frac{d^{2} \bar{U}_{1}}{d x_{2}^{2}} .
$$

As the pressure gradient is specified as a constant, equation (2.10) may be integrated once and rearranged to obtain

$$
\tau\left(x_{2}\right)-v_{0} \bar{U}_{1}=-\overline{u_{1} u_{2}}+\nu \frac{d \bar{U}_{1}}{d x_{2}}-v_{0} \bar{U}_{1}=x_{2} \frac{d \bar{P}}{d x_{1}}+c_{1} .
$$

Here $c_{1}$ is a constant that in the canonical channel flow is defined as $\rho u_{\tau}^{2}$ (Tennekes \& Lumley 1972). Due to different wall conditions of the channel flow with transpiration the wall shear stresses on the blowing and suction walls are different, which brought the necessity to use a local friction velocity in (2.11) rather than a global one.

Profiles of the viscous stress, the Reynolds stress and the convective momentum transport together with the total shear stress will be presented and discussed later.

\subsection{The two-point correlation equations}

The space and time correlation functions in the theory of turbulence was first introduced by Keller \& Friedmann (1924). Various authors derived the complete system of two-point correlation equations (see e.g. Hinze 1959; McComb 1990), while Keller \& Friedmann (1924) were also the first who closed it by writing the third moment via the second moment and the mean. Later it was found that higher order correlations may indeed not be neglected and the infinite number of the multi-point correlation (MPC) equations 
rather than two-point correlation should be taken into account. A first derivation of the full MPC equation may have been derived in Oberlack (2000).

In order to comprehend the question why the two- or multi-point correlation equation need to be employed at all for the finding of turbulent scaling laws we may for a moment consider the case of decaying isotropic turbulence. Here, the one-point equation $\frac{d k}{d t}=-\varepsilon$ does not deliver any information that the kinetic energy $k$ usually may decay according to a power law, if some transitional period has passed. However, the corresponding two-point equation, the von Kármán-Howarth equation (von Kármán \& Howarth 1938), admits a similarity solution as was already shown in the original paper of von Kármán Howarth and, probably unknown to the authors, is an implicit use of Lie symmetries. It delivers the power law decay for the turbulent kinetic energy and at the same time the algebraic growth of the integral length scale. Hence, transferring back to the present problem of a shear flow with transpiration we may conclude that the mean momentum equation (2.10) alone is not sufficient to rigorously derive the requested scaling property.

In the present section we only focus on the two-point correlation (TPC) equations in its most general form

$$
\begin{aligned}
\frac{\overline{\mathrm{D}} R_{i j}}{\overline{\mathrm{D}} t}+ & R_{k j} \frac{\partial \bar{U}_{i}(\boldsymbol{x}, t)}{\partial x_{k}}+\left.R_{i k} \frac{\partial \bar{U}_{j}(\boldsymbol{x}, t)}{\partial x_{k}}\right|_{\boldsymbol{x}+\boldsymbol{r}}+\left[\bar{U}_{k}(\boldsymbol{x}+\boldsymbol{r}, t)-\bar{U}_{k}(\boldsymbol{x}, t)\right] \frac{\partial R_{i j}}{\partial r_{k}} \\
& +\frac{\partial \overline{p u}_{j}}{\partial x_{i}}-\frac{\partial \overline{p u}_{j}}{\partial r_{i}}+\frac{\partial \overline{u_{i} p}}{\partial r_{j}}-\nu\left[\frac{\partial^{2} R_{i j}}{\partial x_{k} \partial x_{k}}-2 \frac{\partial^{2} R_{i j}}{\partial x_{k} \partial r_{k}}+2 \frac{\partial^{2} R_{i j}}{\partial r_{k} \partial r_{k}}\right] \\
& +\frac{\partial R_{(i k) j}}{\partial x_{k}}-\frac{\partial}{\partial r_{k}}\left[R_{(i k) j}-R_{i(j k)}\right]=0,
\end{aligned}
$$

without introducing any closure, where the second and third order correlation tensors are defined as

$$
\begin{aligned}
& \left.R_{i j}(\boldsymbol{x}, \boldsymbol{r} ; t)=\overline{u_{i}(\boldsymbol{x}, t) u_{j}(\boldsymbol{x}+\boldsymbol{r}, t}\right), \overline{p u}_{j}=\overline{p(\boldsymbol{x}, t) u_{j}(\boldsymbol{x}+\boldsymbol{r}, t)}, \overline{u_{i} p}=\overline{u_{i}(\boldsymbol{x}, t) p(\boldsymbol{x}+\boldsymbol{r}, t)}, \\
& \left.\left.R_{(i k) j}(\boldsymbol{x}, \boldsymbol{r} ; t)=\overline{u_{i}(\boldsymbol{x}, t) u_{k}(\boldsymbol{x}, t) u_{j}(\boldsymbol{x}+\boldsymbol{r}, t}\right), R_{i(j k)}(\boldsymbol{x}, \boldsymbol{r} ; t)=\overline{u_{i}(\boldsymbol{x}, t) u_{j}(\boldsymbol{x}+\boldsymbol{r}, t) u_{k}(\boldsymbol{x}+\boldsymbol{r}, t}\right) . \\
& \text { and } \overline{\overline{\mathrm{D}} t}=\left(\frac{\partial}{\partial t}+\bar{U}_{k} \frac{\partial}{\partial x_{k}}\right) \text { is the mean substantial derivative. Continuity equations for }
\end{aligned}
$$
the TPC have the following form

$$
\frac{\partial R_{i j}}{\partial x_{i}}-\frac{\partial R_{i j}}{\partial r_{i}}=0, \quad \frac{\partial R_{i j}}{\partial r_{j}}=0
$$

and

$$
\frac{\partial \overline{p u}_{i}}{\partial r_{i}}=0, \quad \frac{\partial \overline{u_{j} p}}{\partial x_{j}}-\frac{\partial \overline{u_{j} p}}{\partial r_{j}}=0 .
$$

Equations for higher order correlations have a rather similar form and may be taken from Oberlack (2000). In the present study Lie group analysis is used to find symmetry transformations and in turn self-similar solutions of the TPC equations. For the present flow the TPC equations (2.12) reduce to the following form

$$
\begin{aligned}
\bar{U}_{2} \frac{\partial R_{i j}}{\partial x_{2}}+ & R_{2 j} \delta_{i 1} \frac{\partial \bar{U}_{1}\left(x_{2}\right)}{\partial x_{2}}+\left.R_{i 2} \delta_{1 j} \frac{\partial \bar{U}_{1}\left(x_{2}\right)}{\partial x_{2}}\right|_{x_{2}+r_{2}}+\frac{\partial \overline{p u}_{j}}{\partial x_{i}}-\frac{\partial \overline{p u}_{j}}{\partial r_{i}} \\
& +\frac{\partial \overline{u_{i} p}}{\partial r_{j}}+\left[\bar{U}_{1}\left(x_{2}+r_{2}\right)-\bar{U}_{1}\left(x_{2}\right)\right] \frac{\partial R_{i j}}{\partial r_{1}}-\nu\left[\frac{\partial^{2} R_{i j}}{\partial x_{2} \partial x_{2}}-2 \frac{\partial^{2} R_{i j}}{\partial x_{2} \partial r_{2}}+2 \frac{\partial^{2} R_{i j}}{\partial r_{k} \partial r_{k}}\right] \\
& +\frac{\partial R_{(i 2) j}}{\partial x_{2}}-\frac{\partial}{\partial r_{k}}\left[R_{(i k) j}-R_{i(j k)}\right]=0,
\end{aligned}
$$




$\begin{array}{cccccccccccc}R e_{\tau} & v_{0}^{+} & v_{0} / U_{b} & L_{x_{1}} / h & L_{x_{3}} / h & \Delta x_{1}^{+} & \Delta x_{3}^{+} & N_{x_{1}} & N_{x_{2}} & N_{x_{3}} & N_{F} & u_{\tau} T / h \\ 250 & 0 & 0 & 4 \pi & 2 \pi & 4.1 & 6.1 & 768 & 251 & 256 & 48 & 100 \\ 250 & 0.05 & 0.003 & 4 \pi & 2 \pi & 4.1 & 6.1 & 768 & 251 & 256 & 161 & 150 \\ 250 & 0.1 & 0.0069 & 4 \pi & 2 \pi & 4.2 & 6.1 & 768 & 251 & 256 & 197 & 70 \\ 250 & 0.16 & 0.0164 & 4 \pi & 2 \pi & 4.2 & 6.2 & 768 & 251 & 256 & 161 & 120 \\ 250 & 0.26 & 0.05 & 4 \pi & 2 \pi & 4.2 & 6.2 & 768 & 251 & 256 & 136 & 150 \\ & & & & & & & & & & & \\ 480 & 0 & 0 & 8 \pi & 6 \pi & 15.3 & 11.7 & 768 & 385 & 768 & 95 & 31 \\ 480 & 0.05 & 0.003 & 8 \pi & 6 \pi & 15.6 & 11.7 & 768 & 385 & 768 & 226 & 41 \\ 480 & 0.1 & 0.0075 & 8 \pi & 6 \pi & 15.6 & 11.7 & 768 & 385 & 768 & 310 & 40 \\ 480 & 0.16 & 0.0164 & 8 \pi & 6 \pi & 15.7 & 11.8 & 768 & 385 & 768 & 212 & 25 \\ 480 & 0.26 & 0.049 & 8 \pi & 6 \pi & 15.8 & 11.8 & 768 & 385 & 768 & 214 & 30 \\ & & & & & & & & & & & \\ 850 & 0.05 & 0.0026 & 8 \pi & 6 \pi & 6.8 & 5.1 & 3072 & 471 & 3072 & 160 & 26 \\ 850 & 0.16 & 0.016 & 8 \pi & 6 \pi & 7 & 5.2 & 3072 & 471 & 3072 & 205 & 22\end{array}$

TABLE 1. Summary of the simulations. $v_{0}^{+}=v_{0} / u_{\tau}$ is the transpiration rate. $L_{x_{1}}, L_{x_{3}}, \Delta x_{1}^{+}$ and $\Delta x_{3}^{+}$are respectively the periodic dimensions of the numerical box and the resolutions in streamwise and spanwise directions. $N_{x_{1}}, N_{x_{2}}, N_{x_{3}}$ are numbers of collocation points in streamwise, wall-normal and spanwise directions, correspondingly. $N_{F}$ is the number of accumulated statistical fields. $T$ is the computational time spanned by those fields.

since $\bar{U}_{i}\left(x_{2}\right)=\left(\bar{U}_{1}\left(x_{2}\right), \bar{U}_{2}, 0\right)$ and, we recall, $\bar{U}_{2}=v_{0}$.

In this context a natural question arises as to why it is sufficient to employ the mean momentum and the two-point correlation equation for the subsequent Lie symmetry analysis without taking into account the entire infinite set of MPC equations. Here, the deeper reason rests on the fact, that correlation equations have only a coupling to its direct neighboring equation of the next higher tensor order. Hence, without giving a proof for this, it is sufficient to verify that the derived symmetries for a given correlation tensor order are consistent to the next higher order. For the present case which relies on relatively simple symmetries this may readily be verified.

\subsection{DNS of the channel flow with wall blowing and suction}

In order to verify the scaling laws to be obtained for the different regions of the flow in the sections to follow we conduct a number of DNS for different transpiration rates and Reynolds numbers.

For the present DNS we employ a numerical code developed at the School of Aeronautics, Technical University of Madrid (Hoyas \& Jiménez 2006). The code solves the Navier-Stokes equations for an incompressible fluid in velocity-vorticity formulation (see e.g. Kim et al. 1987). In the streamwise and spanwise directions $\left(x_{1}, x_{3}\right)$ Fourier discretization is used. In the wall-normal direction, $\left(x_{2}\right)$, a seven-point compact finite difference scheme (Lele 1992) is applied. Full details of the numerical methods are given in Hoyas \& Jiménez (2006). The DNS data of Sumitani \& Kasagi (1995) are used for the validation of the code. Validation results are not shown in the present paper, as they were included in Avsarkisov et al. (2011).

Production runs can be divided into three sets depending on the friction Reynolds number $R e_{\tau}$. The first two simulation sets consists of four cases for different transpiration rates, while the highest Reynolds number simulation set consists of only two cases for small and medium transpiration numbers. A complete summary of the flow and the numerical parameters are given in Table 1. Using the DNS and the experimental results of Sumitani \& Kasagi (1995) and Antonia et al. (1986) we concluded that for the smaller 
Reynolds number simulations $\left(R e_{\tau}=250\right)$ it will be sufficient to use a $4 \pi h \times 2 h \times 2 \pi h$ box. A validation of this assumption may be taken from figure 4 , where the results for the two-point cross correlation function $R_{12} / \sqrt{\left(\overline{u_{1} u_{2}}\right)^{2}}$ for $R e_{\tau}=250, v_{0}^{+}=0.16$ are shown. For higher Reynolds numbers we selected a box similar to the one that was used in del Álamo \& Jiménez (2003), del Álamo et al. (2004) and Hoyas \& Jiménez (2006). Due to an increased dissipation at the blowing side we had to use more grid points in wall normal direction than it is usually taken in a classical channel flow DNS to keep the resolution of $\Delta x_{2}=1.8 \eta$ approximately constant in terms of the local Kolmogorov length scale $\eta$.

\section{New scaling laws of the turbulent channel flow with wall transpiration}

As it was mentioned in the introduction, the existence of the uniform transpiration implies an asymmetry not only for the mean velocity profile, but also for the distribution of the stresses. Blowing redistributes Reynolds shear stress into the core region. This effect is similar to the one that is observed in a turbulent Couette-Poiseuille flow, where the position at which $\tau\left(x_{2}\right)=0$ is no longer in the center of the channel in contrast to what is seen in a pure Poiseuille-type flow (see e.g. Nakabayashi et al. 2004). In addition, we observe that suction creates a very high wall-normal gradient of the streamwise velocity in the vicinity of the suction wall. With growing transpiration rate this asymmetry is amplified exhibiting an ever increasing difference in physical properties between the canonical channel flow and the flow with transpiration, as may be taken from the figures 2 and 3 . At moderate transpiration rates, i.e. $u_{\tau} / v_{0}<0.16$, in the vicinity of the blowing side the transpiration velocity produces extra Reynolds shear stress and suppresses the viscous stress, while the reverse is observed on the suction side. At high transpiration rates, i.e. $0.16 \leqslant v_{0} / u_{\tau} \leqslant 0.26$, the Reynolds shear stress vanishes on the suction side, while the viscous stress disappears on the blowing one, as it can be taken from the figures $2(d, e)$ and $3(d, e)$. The flow in the near-wall region is rather particular and very different from the classical Poiseuille flow as in this region the transpiration velocity is an order of magnitude smaller than the streamwise velocity. As a result, in contrast to the classical flow, streamlines in the near-wall region are perpendicular to the wall. Further away from the wall they are strongly bent towards the downstream direction. A first theoretical attempt to study turbulent Poiseuille flow at high transpiration rates was made by Vigdorovich \& Oberlack (2008), who used the method of matched asymptotic expansions to show that the skin friction vanishes at the blowing wall, while the point of maximum streamwise velocity approaches the suction wall.

A second critical effect, that can be taken from figures 2 and 3, is the non-coincidence of the points of zero turbulent shear stress with the point of zero viscous stress, which appears at high transpiration rates. Usually located in the center of the channel, as it is shown on the figures $2(b)$ and $3(b)$, i.e. $x_{2}=h$, the point of zero shear stress is slightly shifted towards the blowing wall while the point of maximum velocity moves towards the suction side.

The non-coincidence of the different locations of the two points with $\tau\left(x_{2}\right)=0$ and $\bar{U}_{1 \max }\left(x_{2}\right)$ may not only be induced by transpiration. This effect, however, may also be observed in the turbulent channel flows with asymmetric boundary conditions such as, rough wall/smooth wall conditions (see e.g. Hanjalić \& Launder 1972a), or, generally speaking, when the values of the stresses at the wall $\tau_{w}$ are different on the upper and the lower walls. The exception, when the difference of the stresses at the walls does not induce this non-coincidence of $\tau\left(x_{2}\right)=0$ and $\bar{U}_{1 \max }\left(x_{2}\right)$ may be found in the Couette-Poiseuille 
New Scaling Laws for Turbulent Poiseuille Flow with Wall Transpiration 9
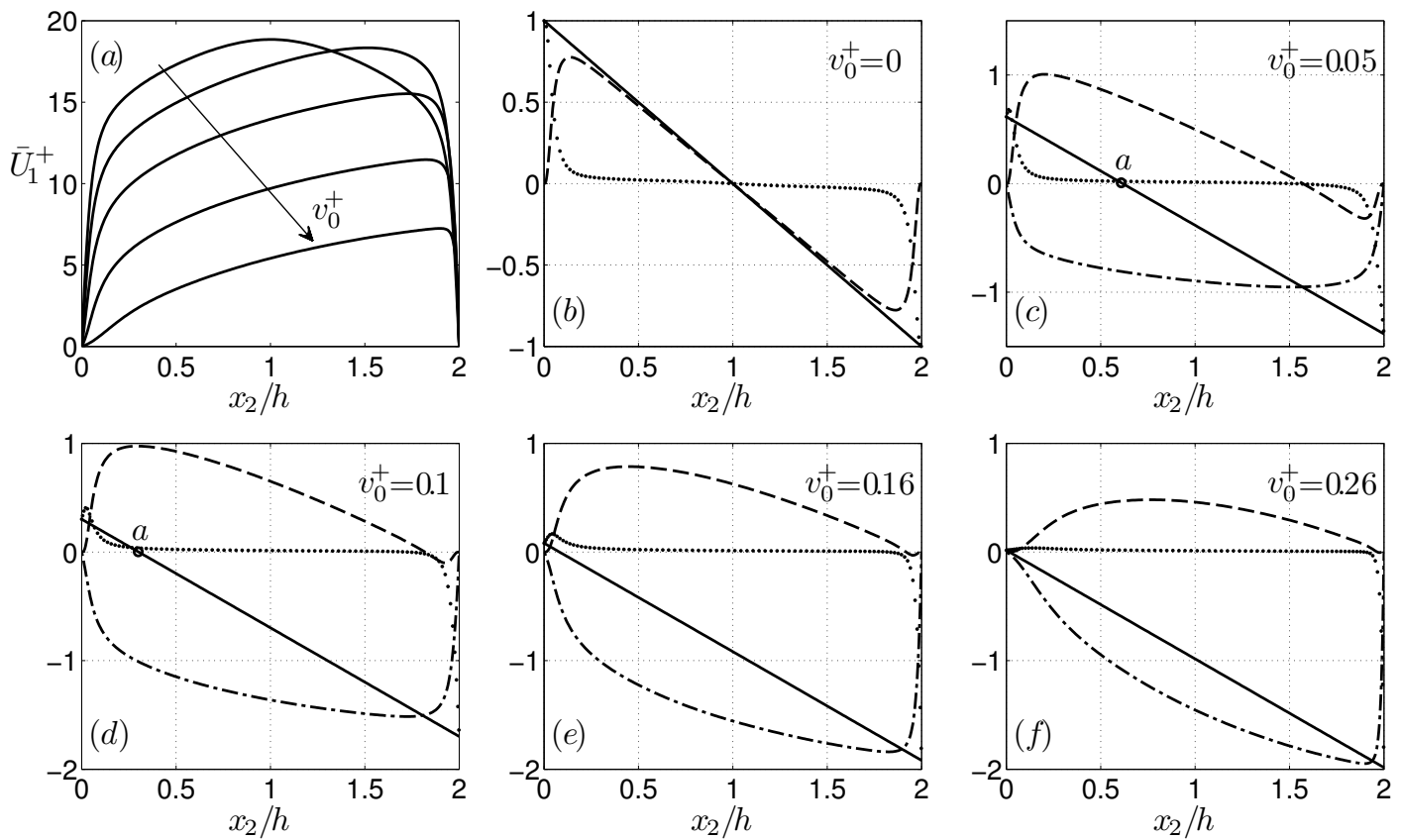

FIGURE 2. Mean velocity and shear stress distribution profiles at $R e_{\tau}=250$ and $v_{0}^{+}=0.0,0.05,0.1,0.16,0.26$. $(a)$ mean velocity profiles. Panels $(b)-(f)$ depict shear stress distributions with increasing transpiration rate. $\frac{d \bar{U}_{1}^{+}}{d x_{2}^{+}}: \cdots ;{\overline{u_{1} u_{2}}}^{+}:--; \tau^{+}-v_{0}^{+} \bar{U}_{1}^{+}:-; v_{0}^{+} \bar{U}_{1}^{+}$: -.- Blowing wall is at $x_{2}=0$ and suction wall is at $x_{2}=2 h$. The point of zero shear stress measured from the blowing wall is denoted by $a$.
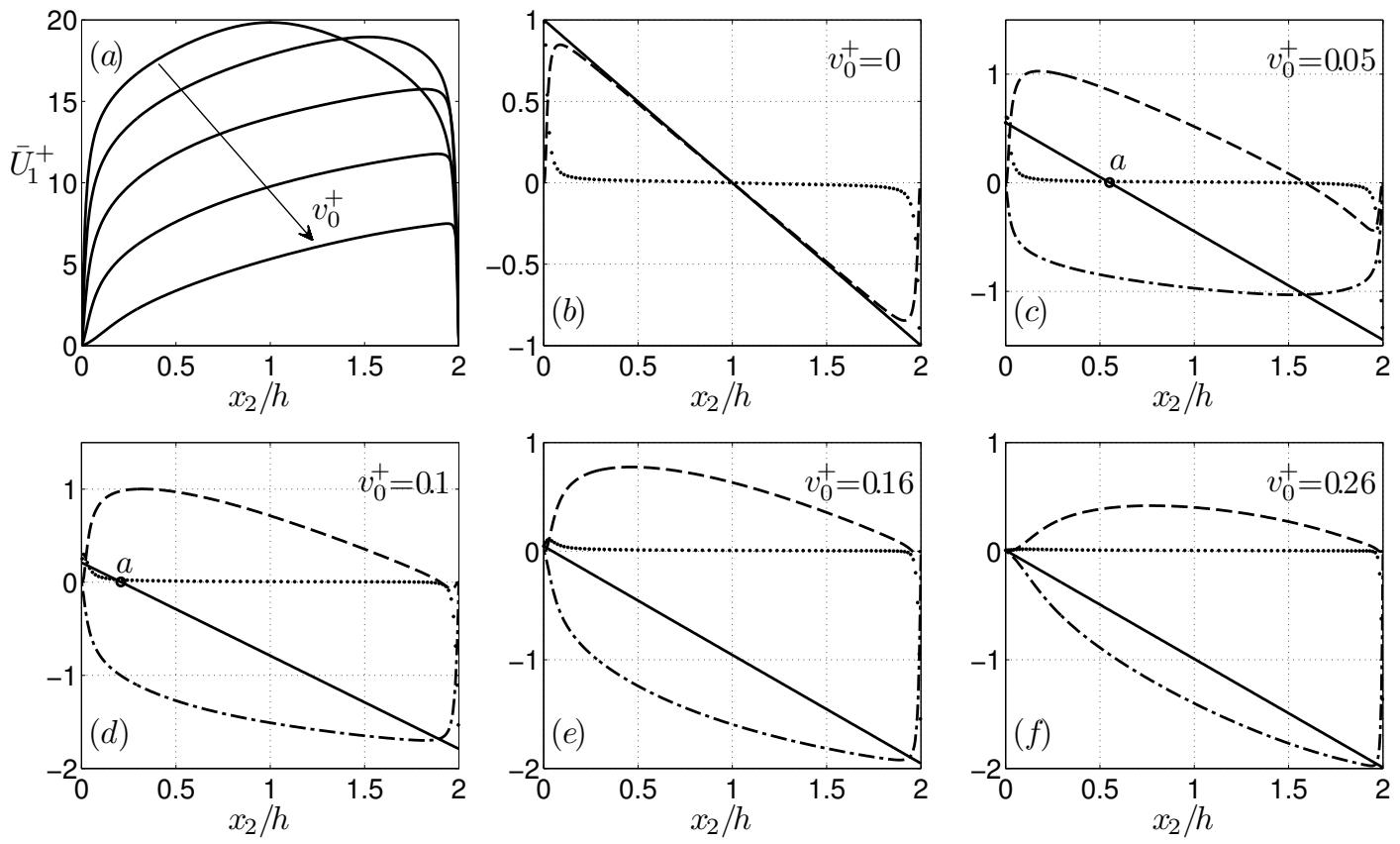

Figure 3. The same as on figure 2 but for $R e_{\tau}=480$. 


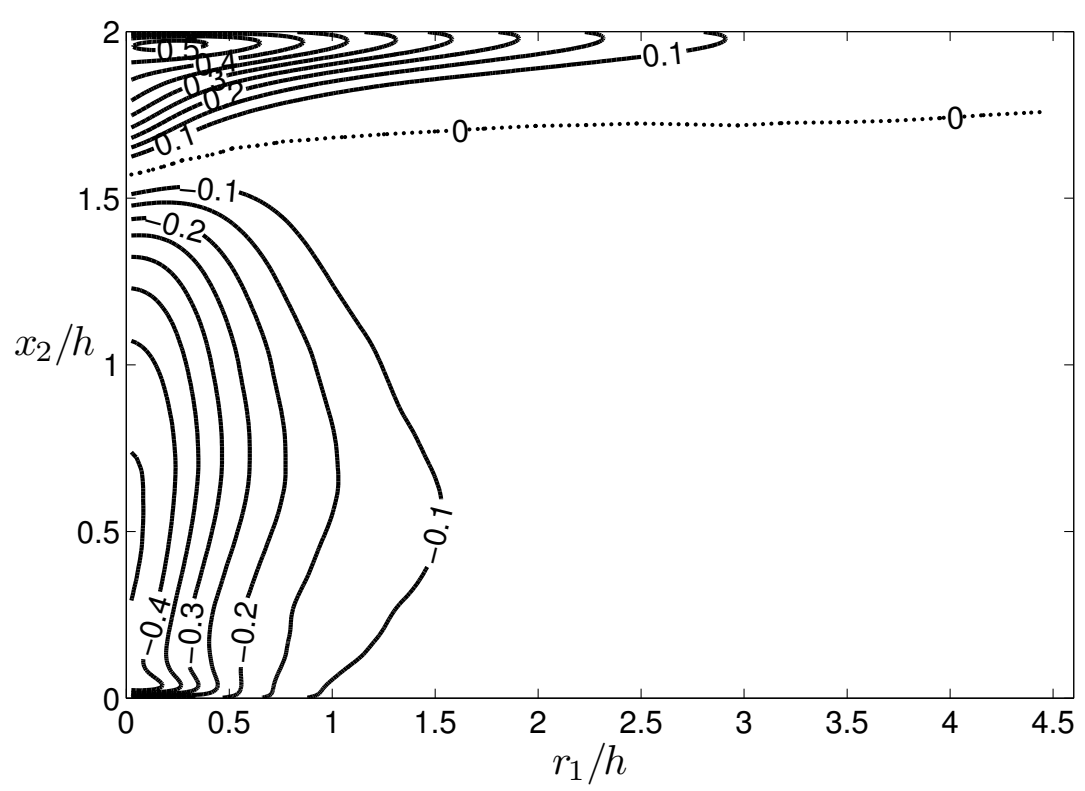

FIgURE 4. Iso-contours of the two-point cross-correlation function normalized on stresses, i.e. $R_{12} / \sqrt{\left(\overline{u_{1} u_{2}}\right)^{2}}$, as a function of the streamwise separation $r_{1} / h$ and the wall-normal coordinate $x_{2} / h$. Further we have $r_{2} / h=r_{3} / h=0$. The spacing of the contour lines 0.05 ; positive/negative; -...., zero.

flow, when there is no region of zero shear stress between the two walls (Couette-type flow) (see e.g. Nakabayashi et al. 2004; Johnstone et al. 2010).

An interesting result was obtained from the analysis of the shear stress combined with the convective term, $\tau^{+}-v_{0}^{+} \bar{U}_{1}^{+}$. The point of the balance of shear stress and convective momentum transport $(a / h)$, approaches the blowing wall as the transpiration increases, that can be taken from the figures 2 and 3. From simple geometrical considerations one may derive the relation

$$
\frac{a}{h}=\frac{\tau_{w b}}{\left(\left|\tau_{w b}\right|+\left|\tau_{w s}\right|\right) / 2},
$$

which indicates that the position of the balance point depends on the values of the both shear stresses at the walls, i.e. $\tau_{w b}$ and $\tau_{w s}$.

Another profound insight into the turbulence characteristics of the present flow type is obtained by analyzing the two-point correlations. It may be taken from figure 4 that close to the suction wall $\left(x_{2} \rightarrow 2 h\right)$ the streamwise two-point cross correlation $R_{12}$ indicates a rather long correlation length as it decays slowly in $r_{1}$-direction. In contrast, on the blowing side $\left(x_{2} \rightarrow 0\right)$ correlations show a considerably shorter extend. That nicely agrees with the structural analysis of the near-wall region conducted by Sumitani \& Kasagi (1995). They showed that near the blowing side the flow is highly populated with small scale coherent vortical structures, while on the suction side coherent vortical structures appear less frequently, however, at significantly larger scales.

Later in this section new scaling laws will be derived for the present turbulent Poiseuille flow with non-zero blowing and suction $\left(0.05<v_{0} / u_{\tau}<0.16\right)$. Already at this point, from the stress distribution displayed in figures 2 and 3, one can predict that at high transpiration rates the near-wall log-law does not exist on the suction side because the Reynolds shear stress in that region is negative or equal to zero and that the viscous 
sublayer at the blowing wall will vanish because viscous stress in the near-blowing-wall region is almost zero.

\subsection{New logarithmic scaling law from Lie symmetries of the correlation equations}

One of the key objectives of the present analysis is to further develop and validate the Lie group theory for the multi-point statistics of turbulence employing the turbulent Poiseuille flow with wall transpiration. The first step to accomplish this objective is to find symmetry transformations which do not change the form of the TPC equation (2.15). The application of these symmetry groups will later facilitate finding a group invariant solution of the TPC equation in fluid mechanics often of self-similar type. Finally, this leads to the reduction of the TPC equation and, at the same time, determines the functional form of the turbulent scaling law for the mean velocity. The final step of the analysis will be the validation of the new turbulent scaling law by employing the present DNS data.

At this point it is important to note that TPC equations (2.12)-(2.15), which are to be analyzed with respect to its symmetries, have undergone a large Reynolds number asymptotics as was first presented in Oberlack (2000). Therein it is shown that for correlation distances $|r| \leqslant \eta$, where $\eta$ is the Kolmogorov length scale, all viscous terms in the latter equations vanish. Exclusion of all the viscous terms from TPC equations (2.12)-(2.15) allows to recover one scaling symmetry, which was originally lost due to the presence of viscosity.

The starting point of this analysis is the observation that the boundary condition (2.8), in particular for the transpiration velocity, may be symmetry breaking primarily in the core region of the flow. In the present section only an abbreviated approach will be presented, while more mathematical details are available in Oberlack \& Rosteck (2010) and in the Appendix B of Oberlack (2001).

In order to derive a new turbulent scaling law for the present flow from the TPC equation we need to consider the appropriate symmetry transformations. For the present problem it is sufficient to focus on the three scaling groups $\left(\bar{T}_{1}, \bar{T}_{2}, \bar{T}_{s}^{\prime}\right)$, the translation group in space $\left(\bar{T}_{x_{2}}\right)$ and the translation group of the averaged velocity $\left(\bar{T}_{\bar{U}_{i}}\right)$. In global form these transformation groups are defined as:

$$
\begin{aligned}
& \bar{T}_{1}: t^{*}=t, \boldsymbol{x}^{*}=e^{k_{1}} \boldsymbol{x}, \boldsymbol{r}_{(l)}^{*}=e^{k_{1}} \boldsymbol{r}_{(l)}, \bar{U}_{i}^{*}=e^{k_{1}} \bar{U}_{i}, \bar{P}^{*}=e^{2 k_{1}} \bar{P}, \\
& R_{i j}^{*}=e^{2 k_{1}} R_{i j},{\overline{p u_{j}}}^{*}=e^{3 k_{1}} \overline{p u_{j}},{\overline{u_{i} p}}^{*}=e^{3 k_{1}} \overline{u_{i} p}, \ldots, \\
& \bar{T}_{2}: t^{*}=e^{k_{2}} t, \boldsymbol{x}^{*}=\boldsymbol{x}, \boldsymbol{r}_{(l)}^{*}=\boldsymbol{r}_{(l)}, \bar{U}_{i}^{*}=e^{-k_{2}} \bar{U}_{i}, \bar{P}^{*}=e^{-2 k_{2}} \bar{P}, \\
& R_{i j}^{*}=e^{-2 k_{2}} R_{i j},{\overline{p u_{j}}}^{*}=e^{-3 k_{2}} \overline{p u_{j}}, \overline{u_{i} p^{*}}=e^{-3 k_{2}} \overline{u_{i} p}, \ldots, \\
& \bar{T}_{s}^{\prime}: t^{*}=t, \boldsymbol{x}^{*}=\boldsymbol{x}, \boldsymbol{r}_{(l)}^{*}=\boldsymbol{r}_{(l)}, \bar{U}_{i}^{*}=\mathrm{e}^{k_{s}} \bar{U}_{i}, \bar{P}^{*}=e^{k_{s}} \bar{P}, \\
& R_{i j}^{*}=\mathrm{e}^{k_{s}}\left[R_{i j}+\left(1-\mathrm{e}^{k_{s}}\right) \bar{U}_{i} \bar{U}_{j}\right],{\overline{p u_{j}}}^{*}=e^{k_{s}} \overline{p u_{j}}+\left(e^{k_{s}}-e^{2 k_{s}}\right) \bar{P} \bar{U}_{j}, \\
& {\overline{u_{i} p}}^{*}=e^{k_{s}} \overline{u_{i} p}+\left(e^{k_{s}}-e^{2 k_{s}}\right) \bar{P} \bar{U}_{i}, \ldots, \\
& \bar{T}_{x_{2}}: t^{*}=t, \boldsymbol{x}^{*}=\boldsymbol{x}+k_{x_{2}}, \quad \boldsymbol{r}_{(l)}^{*}=\boldsymbol{r}_{(l)}, \bar{U}_{i}^{*}=\bar{U}_{i}, \\
& \bar{P}^{*}=\bar{P}, R_{i j}^{*}=R_{i j},{\overline{p u_{j}}}^{*}=\overline{p u_{j}},{\overline{u_{i} p^{*}}}^{*} \overline{u_{i} p}, \ldots, \\
& \bar{T}_{\bar{U}_{i}}: t^{*}=t, \quad \boldsymbol{x}^{*}=\boldsymbol{x}, \quad \boldsymbol{r}_{(l)}^{*}=\boldsymbol{r}_{(l)}, \bar{U}_{i}^{*}=\bar{U}_{i}+k_{\bar{U}_{i}} \text {, } \\
& \bar{P}^{*}=\bar{P}, \quad R_{i j}^{*}=R_{i j},{\overline{p u_{j}}}^{*}=\overline{p u_{j}},{\overline{u_{i} p^{*}}}^{*} \overline{u_{i} p}, \ldots .
\end{aligned}
$$

Above and also further down, the dots denote the fact that in principle higher order correlations are part of the symmetry transformation since the full MPC equation is 
infinite dimensional. This, however, will not be considered presently and we only focus on the mean velocity and the TPC.

The first two scaling symmetries are well-known from the Euler and the Navier-Stokes equations describing scaling of space and time. The third one is a rather new group and independent of (3.2) and (3.3). It represents the scaling of all TPC or MPC tensors, and it is a purely statistical property of these equations (Oberlack \& Rosteck 2010). In fact it is a property of all linear equations. One of the most crucial symmetries for the results to follow and also a key ingredient of the classical log-law (Oberlack 2001) is symmetry (3.6). It is also of purely statistical nature and was discovered in the context of an infinite set of statistical symmetries in (Oberlack \& Rosteck 2010). It is noted that the first hint towards (3.6) has been given by Kraichnan (1965).

In local (infinitesimal) form the symmetries (3.2)-(3.6) are given by

$$
\begin{aligned}
\overline{\mathrm{X}}_{1}= & x_{2} \frac{\partial}{\partial x_{2}}+\bar{U}_{i} \frac{\partial}{\partial \bar{U}_{i}}+r_{i} \frac{\partial}{\partial r_{i}}+2 R_{i j} \frac{\partial}{\partial R_{i j}}+3 \overline{p u_{j}} \frac{\partial}{\partial \overline{p u_{j}}}+3 \overline{u_{i} p} \frac{\partial}{\partial \overline{u_{i} p}}+\ldots \\
\overline{\mathrm{X}}_{2}= & t \frac{\partial}{\partial t}-\bar{U}_{i} \frac{\partial}{\partial \bar{U}_{i}}-2 R_{i j} \frac{\partial}{\partial R_{i j}}-3 \overline{p u_{j}} \frac{\partial}{\partial \overline{p u_{j}}}-3 \overline{u_{i} p} \frac{\partial}{\partial \overline{u_{i} p}}+\ldots \\
\overline{\mathrm{X}}_{s}= & \bar{U}_{i} \frac{\partial}{\partial \bar{U}_{i}}+\left(R_{i j}-\bar{U}_{i} \bar{U}_{j}\right) \frac{\partial}{\partial R_{i j}}+\left(\overline{p u_{j}}-\bar{P} \bar{U}_{j}\right) \frac{\partial}{\partial \overline{p u_{j}}}+ \\
& \left(\overline{u_{i} p}-\bar{U}_{i} \bar{P}\right) \frac{\partial}{\partial \overline{u_{i} p}}+\ldots \\
\overline{\mathrm{X}}_{x_{2}}= & \frac{\partial}{\partial x_{2}}, \\
\overline{\mathrm{X}}_{\bar{U}_{i}}= & \frac{\partial}{\partial \bar{U}_{i}}+\ldots .
\end{aligned}
$$

As the latter groups are linearly independent, any linear combination of the symmetries (3.7)-(3.11), yields a new multi-parameter group which is also a symmetry of the TPC equation. From the latter we may finally construct the invariant surface condition (see e.g. Bluman et al. 2010) encompassing all groups given above

$$
\frac{\mathrm{d} x_{2}}{k_{1} x_{2}+k_{x_{2}}}=\frac{\mathrm{d} r_{(k)}}{k_{1} r_{(k)}}=\frac{\mathrm{d} \bar{U}_{i}}{\left(k_{1}-k_{2}+k_{s}\right) \bar{U}_{i}+k_{\bar{U}_{i}}}=\cdots,
$$

where in the present paper any further invariance conditions for higher correlations are omitted.

At this point, none of the group parameters $k_{i}$ in (3.12) are determined. In order to determine at least some of them we may invoke the condition (2.9), i.e. $\bar{U}_{2}=v_{0}$, as this is the key influencing factor for altering the turbulent Poiseuille flow. As it acts primarily on the velocity we consider the concatenated global transformations for the mean velocity

$$
\bar{U}_{i}^{*}=e^{k_{1}-k_{2}+k_{s}} \bar{U}_{i}
$$

taken from (3.2)-(3.4) and, for the moment, omit any other part of the transformations. In order to comprehend the following, we may first recall that invariance, and in turn invariant reduction, requires a knowledge of the symmetries admitted by the underlying equation, here the TPC equation (2.15). In a second step, however, for the construction of a concrete solution, symmetries, or a combination of them, have to be consistent to the imposed boundary conditions. Presently this means (3.13) has to be conformal to (2.9) which, after implementing of the former into the latter, leads to

$$
e^{-\left(k_{1}-k_{2}+k_{s}\right)} \bar{U}_{2}^{*}=v_{0} .
$$


As the definition of symmetry implies form invariance, also for the boundary conditions, this provides the constraint

$$
k_{1}-k_{2}+k_{s}=0 .
$$

We may conclude that the transpiration velocity $\left(v_{0}\right)$ is symmetry breaking, most likely in the core region of the flow but also in the near-wall region.

Imposing the latter constraint onto (3.12) and integrating the first with the third term leads to a new logarithmic scaling law for the streamwise mean velocity in the core region

$$
\bar{U}_{1}=A_{1} \ln \left(\frac{x_{2}}{h}+B_{1}\right)+C_{1},
$$

where $A_{1}=k_{\bar{U}_{1}} / k_{1}$ and $B_{1}=k_{x_{2}} / k_{1}$ and hence, they are either functions of the group parameters $k_{i}$ or simply constants of integration as $C_{1}$. If it may be presumed that $v_{0}$ is sufficiently large $\left(0.05 \lesssim v_{0}^{+}\right)$we will subsequently show that the latter new log-law is valid in the core region of a turbulent channel flow with wall transpiration.

For the wall-normal component of the mean velocity $\bar{U}_{2}$ a result similar to (3.16) is obtained from (3.12). Taking into account the additional constraint $k_{\bar{U}_{2}}=0$, we obtain $\bar{U}_{2}\left(x_{2}\right)=C_{2}$, which nicely confirms to $(2.9)$ that the wall-normal component of mean velocity is a constant and is equal to the transpiration velocity $v_{0}$.

\subsection{New viscous sublayer velocity scaling law for the blowing wall}

As was mentioned above, the mean flow momentum equation (2.10) is integrable once leading to (2.11). For a local analysis we may in principle rewrite the momentum equation for each wall and later normalize it on the respective local friction velocities. Presently, however, we will follow a slightly different route and may first reformulate the local friction velocities according to (2.1) and (3.1) by the averaged friction velocity $u_{\tau}$ which is related to the streamwise pressure gradient

$$
u_{\tau b}^{2}=\frac{a}{h} u_{\tau}^{2}, u_{\tau s}^{2}=\frac{2 h-a}{h} u_{\tau}^{2} .
$$

The coefficients $a / h$ and $(2 h-a) / h$ represent the relations $\tau_{w b} / \tau_{w}$ and $\tau_{w s} / \tau_{w}$ respectively defined by (3.1), where $a$ is a parameter that depends on the transpiration velocity. This facilitates a normalization of the terms of the momentum equation with $u_{\tau}$ rather than with local ones, which allows us to directly compare the scaling of the blowing and the suction wall based on the same scaling. The mean momentum equation (2.11) in integrated form for the blowing side and rewritten in non-dimensional form based on $u_{\tau}$ and $\nu$ yields

$$
\frac{d \bar{U}_{1}^{+}}{d x_{2}^{+}}-v_{0}^{+} \bar{U}_{1}^{+}-\overline{u_{1} u_{2}}+=\frac{a}{h}-\frac{x_{2}^{+}}{R e_{\tau}} .
$$

Extending the usual universal near-wall region located in the vicinity of the wall where $x_{2}^{+}$is the wall-normal coordinate and taking the limit $R e_{\tau} \rightarrow \infty$, with $x_{2}^{+}=\mathcal{O}(1)$, the equation $(3.18)$ reduces to

$$
\frac{d \bar{U}_{1}^{+}}{d x_{2}^{+}}-v_{0}^{+} \bar{U}_{1}^{+}-{\overline{u_{1} u_{2}}}^{+}=\frac{a}{h} .
$$

Finally, for the viscous sublayer at the blowing wall, we take the limit $x_{2}^{+} \rightarrow 0$ to obtain ${\overline{u_{1} u_{2}}}^{+} \rightarrow 0$ and $v_{0}^{+} \bar{U}_{1}^{+} \rightarrow 0$ and hence linear velocity scaling law for the blowing side results

$$
\bar{U}_{1}^{+}=\frac{a}{h} x_{2}^{+}
$$




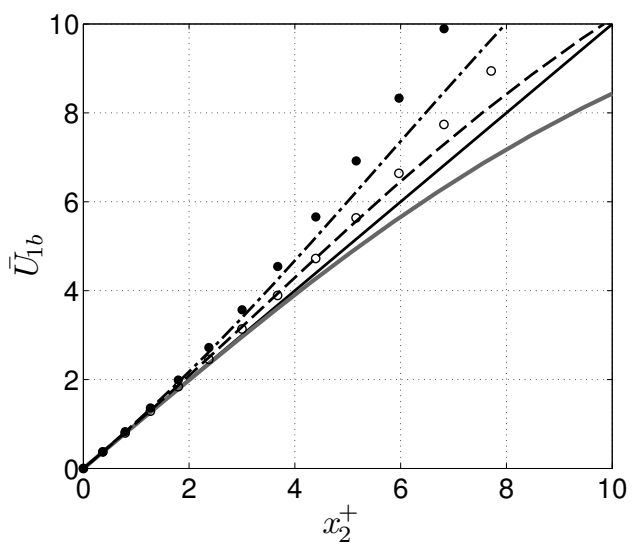

Figure 5. Mean velocity profiles and linear law at the blowing wall. - , linear laws (3.20); $\longrightarrow, v_{0}^{+}=0 ;---, v_{0}^{+}=0.05 ;-.-, v_{0}^{+}=0.1 ; \bullet, v_{0}^{+}=0.16 ;$ o, $v_{0}^{+}=0.26$.

or in rescaled form

$$
\bar{U}_{1 b}=x_{2}^{+}, \quad \text { where } \quad \bar{U}_{1 b}=\frac{\bar{U}_{1}}{u_{\tau b}} \frac{u_{\tau}}{u_{\tau b}}=\bar{U}_{1 b}^{+} u_{\tau b}^{+} .
$$

We note that the true local shear stress derives from the pre-factor on the right hand side of the equation (3.20) and may be re-formulated to give a local shear stress based scaling law employing equation (3.17). Since in the classical Poiseuille flow without transpiration the point of zero shear stress coincides with the centreline of the flow, i.e. $a=h$, we observe that the modified scaling law (3.20) recovers the classical one for $v_{0}=0$.

In the presence of the convective momentum transport term $v_{0} \bar{U}_{1}$, the blowing shifts the viscous shear stress away from the wall towards the channel center as can be taken from figures $2(d-f)$ and $3(d-f)$. Since the viscous stress at the blowing wall is smaller than in a channel with impermeable boundaries, the viscous sublayer at the blowing side is thinner than for the classical flow. At very high transpiration rates $\left(v_{0} / u_{\tau}=0.26\right)$, when the local friction velocity becomes smaller than transpiration velocity $\left(v_{0} / u_{\tau b}=2.9\right)$, blowing creates a strong wall-normal flow in the vicinity of the blowing wall, and we see an increased validity of the linear scaling for $\bar{U}_{1}^{+}$as it is obtained in figure 5 . However, at such a high transpiration rate the viscous stress at the blowing wall is very small even at high Reynolds numbers, i.e. the streamwise velocity gradient in wall-normal direction is small, as can be taken from figures $2(f)$ and $3(f)$.

\subsection{New viscous sublayer velocity scaling law for the suction wall}

A similar analysis of the viscous sublayer on the suction side is not valid as the flow in that region may not be fully turbulent for large transpiration rates. However, in the limit $\overline{u_{1} u_{2}} \rightarrow 0$ it is possible to find the velocity profile by integration of the mean momentum equation (2.10). In its final non-dimensional form the solution may be written as follows

$$
\bar{U}_{1 s}=\frac{2 R e_{v_{0}} e^{2 R e_{v_{0}}}}{1-e^{2 R e_{v_{0}}\left(1-2 R e_{v_{0}}\right)}}\left(1-e^{-x_{2 s}}+\frac{1-e^{2 R e_{v_{0}}}}{2 R e_{v_{0}} e^{2 R e_{v_{0}}}} x_{2 s}\right),
$$

where dimensionless variables in the near-suction-wall scaling have the following forms:

$$
x_{2 s}=\frac{x_{2} v_{0}}{\nu}, \quad \bar{U}_{1 s}=\frac{\bar{U}_{1}}{u_{\tau s}} \frac{v_{0}}{u_{\tau s}}=\bar{U}_{1 s}^{+} v_{0 s}^{+}, \quad R e_{v_{0}}=R e_{\tau s} v_{0 s}^{+}=\frac{h v_{0}}{\nu} .
$$



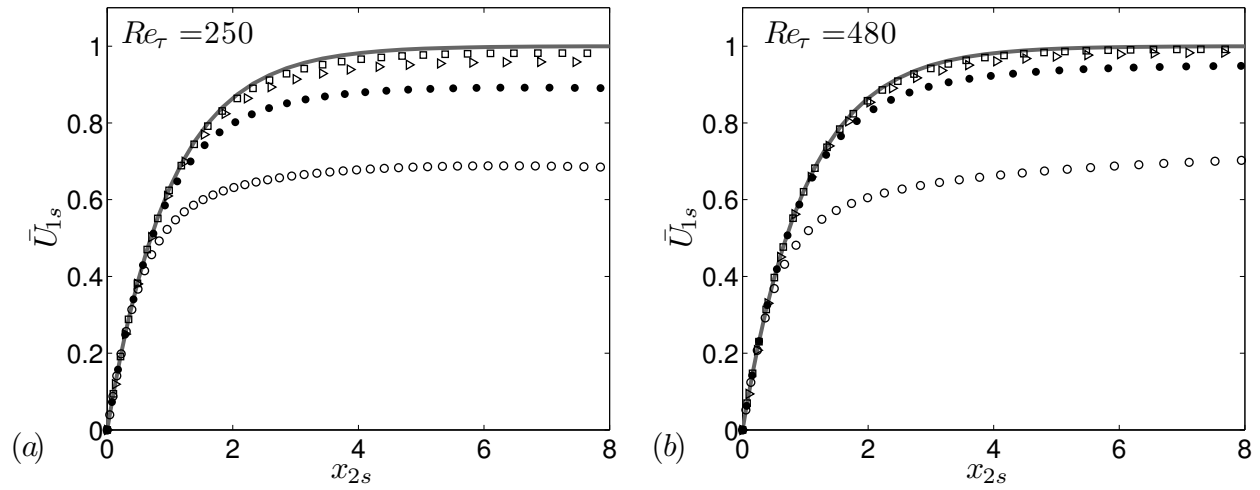

FIGURE 6. Mean velocity profiles and the asymptotic suction profile (3.24) at the suction wall at (a) $R e_{\tau}=250$ and (b) $R e_{\tau}=480$. profile $(3.24) ; \circ, v_{0}^{+}=0.05 ; \bullet, v_{0}^{+}=0.10 ; \Delta$, $v_{0}^{+}=0.16 ; \square, v_{0}^{+}=0.26$.

Note, that $x_{2 s}$ is the wall-normal coordinate, i.e. pointing in a different direction as in the rest of this paper, thus $x_{2 s}$ has been replaced by $2 R e_{\tau s}-x_{2 s}$. Taking the limit $R e_{v_{0}} \rightarrow \infty$, with $x_{2 s}=\mathcal{O}(1),(3.22)$ reduces to the asymptotic suction profile (Griffith \& Meredith 1936; Drazin \& Riley 2006)

$$
\bar{U}_{1 s}=1-e^{-x_{2 s}} .
$$

In application to the turbulent wall-bounded flows with suction the near-wall solution (3.24) may have been obtained first by Tennekes (1965). However, due to too wide intervals between velocity traverses in his experiments velocity profiles did not coincide with the analytical solution (3.24). Presently, we verified (3.24) with the DNS, and the results are presented on figure $6(a, b)$. As it can be taken from figure $6(a, b)$, the mean velocity profiles at very high transpiration rates scales with equation (3.24) much better than at low or moderate transpiration rates. It is also important to note that with increasing $R e_{\tau}$ the domain of agreement between the data and (3.24) increases.

\subsection{Near-wall logarithmic scaling law}

The classical formulation of the near-wall log-law traces back to the seminal work of von Kármán (1930). Later Millikan (1939) employed matched asymptotics to express the logarithmic law of the wall as an overlap region. Presently we reconsider a derivation technique based on Lie group analysis, as it was used in subsection 3.1 to find the log-law in the core region of the channel flow with wall transpiration.

It was first shown in Oberlack (2001) that the near-wall log-law has its roots in Lie symmetries based on a combination of statistical and fluctuating equations. Its first derivation based on the two- and multi-point correlation equations and in particular recognizing the importance of the statistical groups (3.9), (3.11) was obtained in Oberlack $\&$ Rosteck (2010). The authors used the infinite set of MPC equations to derive the nearwall log-law

$$
\bar{U}_{1}^{+}=\frac{1}{\kappa} \ln \left(x_{2}^{+}+A^{+}\right)+C,
$$

and showed that the friction velocity $u_{\tau}$ is the key symmetry breaking parameter in the near-wall region. The latter is a slightly generalized functional form of the usual near-wall scaling law, as it implies the offset $A^{+}$as a displacement height which gives an extended fit of (3.25) to the experimental data (see e.g. Lindgren et al. 2004) and further appears, for example, in the log-law for rough-wall-bounded flows (Jackson 1981), in the overlap 
formulation for the turbulent channel and pipe flows proposed by Wosnik et al. (2000). Recently, similar formulation of the near-wall log-law has been identified by Fife et al. (2009) (also see Klewicki 2013).

An apparent question arises how equation (3.25) may change under the influence of the wall-transpiration. In the present flow configuration the friction velocity $u_{\tau}$ and the transpiration velocity are of the same dimension and, to be shown below, are closely correlated. Further, as both are symmetry breaking for the velocity scaling a functional form of the near-wall log-law obtained from Lie group analysis should be similar to the case without transpiration (3.25).

It was shown above that for the viscous sublayers on each wall the local friction velocity is the key normalization parameter in the equations (3.20) and (3.22), as would be naturally expected. This, however, does not appear to be true for the near-wall log-law. The collapse of the near-wall data appears to be due to the mean friction velocity $u_{\tau}$, which is a measure of the pressure gradient. Similar normalization parameter was used by Telbany \& Reynolds (1981), which suggested that an effective friction velocity, i.e. the one that combines the shear stress information on both walls, should be used, and by Wei et al. (2007), which used a mean friction velocity approach in their analysis of turbulent Couette-Poiseuille flow. This, however, is very different from what was shown in Nikitin \& Pavel'ev (1998). They employed a local $u_{\tau}$ on the blowing wall and observed that the usual von Kármán constant $\kappa$ varies with the blowing velocity.

In order to find the modified constants for the classical near-wall log-law in equation (3.25) due to wall transpiration we may adopt the classical notation given in Millikan (1939). Since blowing increases turbulence it is preferable to derive unaltered near-wall log-law for this region.

According to the results obtained by Jiménez et al. (2001) for the channel flow with permeable boundaries, transpiration only affects the additive constant $C$ of the log-law, while von Kármán constant $\kappa$ is largely unaltered in the logarithmic region if a global $u_{\tau}$ is used. As it will be shown below, the present DNS seem to support the latter finding and equation (3.25) may be rewritten in the following form

$$
\bar{U}_{1}^{+}=\frac{1}{\kappa} \ln \left(x_{2}^{+}+A^{+}\right)+C+C_{1}\left(\frac{v_{0}}{u_{\tau}}\right),
$$

keeping in mind how it was normalized and the function $C_{1}$ vanishes for vanishing $v_{0}$. Here $\kappa$ and $C$ are independent of $v_{0}$ and hence are universal constants obtained for the case without transpiration and based on the global $u_{\tau}$.

As no first principle idea is known to determine $C_{1}\left(v_{0} / u_{\tau}\right)$ we employ a simple curve fitting procedure to fit the new additive function $C_{1}$ which comes down to the following power-law

$$
C_{1}\left(v_{0}^{+}\right)=\alpha\left(\frac{v_{0}}{u_{\tau}}\right)^{\beta},
$$

where $\alpha=-90.62$ and $\beta=1.188$.

The results from the DNS and the modified log-law calculated from equation (3.26) with $A^{+}=0$ are compared in figure 7 . We observe that the near-wall log-law appears to be valid only in the near-wall region on the blowing side, where transpiration increase the Reynolds stress. It was found that the log-region is formed only at small $\left(v_{0}^{+}<\right.$ $0.1)$ transpiration numbers, while at high transpiration rates $\left(v_{0}^{+} \geqslant 0.16\right)$ it seems to disappear. An indicator function shown on figure $9(e, f)$ gives a weak hint toward this conclusion and further confirms the known result that the von Kármán constant $\kappa$ is very sensitive to the Reynolds number and a flat region is almost invisible for small 


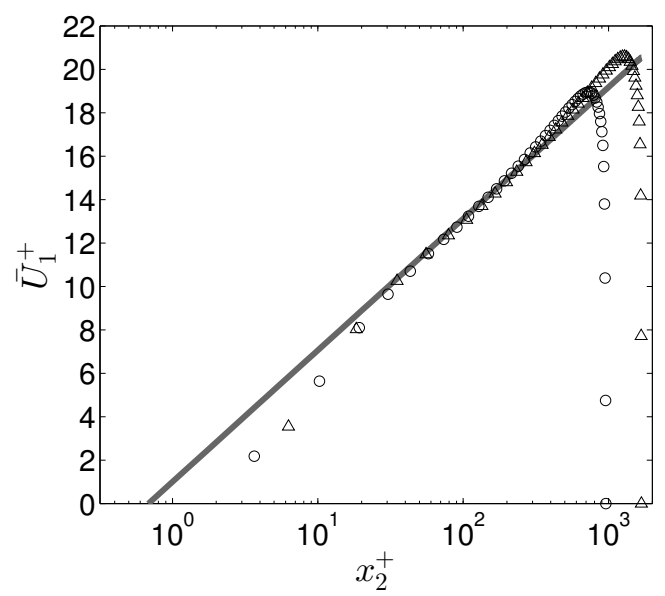

FiguRE 7. Mean velocity profiles of a turbulent channel flow with wall transpiration at the blowing wall. Solid gray line - corresponds to the log-law (3.26), where $\kappa=0.38, A^{+}=0$; , $R e_{\tau}=480 ; \triangle, R e_{\tau}=850$.

Reynolds numbers. Presently it is difficult to make any estimates about the near-wall log-law scaling region and the value of the von Kármán constant as much higher Reynolds number simulations are required.

\section{New logarithmic scaling law of the channel center}

The scaling law (3.16) obtained using Lie symmetry method contains constants $A_{1}, B_{1}$ and $C_{1}$, that cannot be obtained using Lie group analysis alone. For this reason one of the main aim of the present study is to determine the constants employing the DNS results. An open question that arises immediately is a desired velocity scale, that will induce a collapse of the data in the considered region. Form invariance property of the symmetries has proven previously the assumption that transpiration velocity $v_{0}$ is a symmetry breaking constraint in the core region of the flow.

From this one may expect that $v_{0}$ is the appropriate velocity scale for the core region log-law (3.16). However, a changing of $v_{0} / U_{B}$ also significantly changes $u_{\tau} / U_{B}$ as can be taken from figure 8 , and hence the proper velocity scale is not obvious in the first place. From dimensional reasons, the ratio of two velocities result in two non-dimensional groups with a unique functional relation

$$
\frac{u_{\tau}}{U_{B}}=F\left(\frac{v_{0}}{U_{B}}\right) .
$$

The best fit to all DNS data is obtained if instead of $v_{0}$ we invoke $u_{\tau}$ as the appropriate velocity scale. We recall that $u_{\tau}$ is a measure of the pressure gradient as the local $u_{\tau b}$ and $u_{\tau s}$ on each wall are very different. The analysis of the DNS results together with the employment of $u_{\tau}$ as a scaling velocity for $A_{1}$ leads to the fact that the overall scaling appears rather insensitive to the Reynolds numbers and the relative transpiration rates. The latter rescaling leads to $A_{1}=u_{\tau} / \gamma$, where $\gamma=0.3$ has been taken from the DNS data. Note that this is not the usual von Kármán constant $\kappa$.

The offset $B_{1}$ in the equation (3.16) was found to be a very small number. Further, a systematic and careful analysis of the best fitted values did not reveal a unique picture. Hence, $B_{1}$ has been set to zero for all cases, though a better fit of the DNS data to equation (3.16) may be obtained from a non-zero $B_{1}$. 


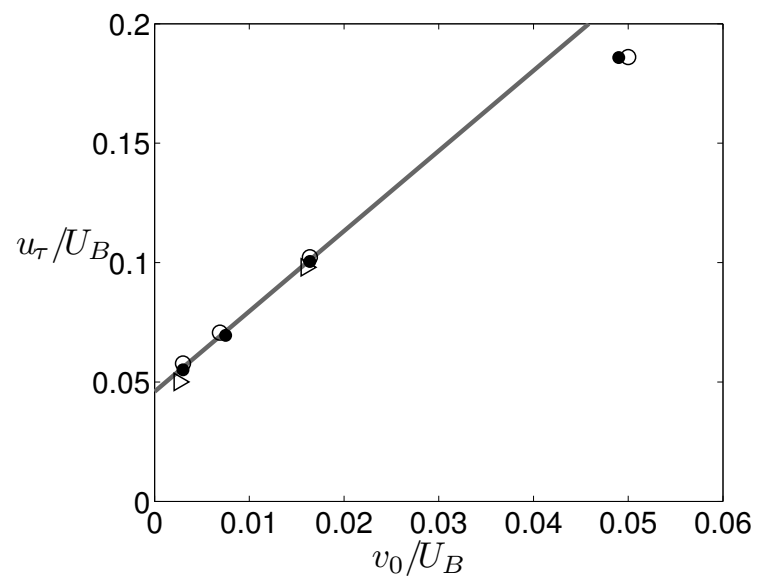

FigURE 8. Relation between transpiration velocity and friction velocity. $\circ, R e_{\tau}=250$ $R e_{\tau}=480 ; \triangleright, R e_{\tau}=850 ;=$, linear relation between $v_{0}$ and $u_{\tau}: u_{\tau}=3.36 v_{0}+0.046$.

Since the new log-law is located in the center of the channel, our present knowledge of turbulent scaling laws suggests a defect type of scaling. Hence, a second global velocity scale is needed to determine $C_{1}$.

In turbulent boundary layer flows we use the free stream velocity $U_{\infty}$ while in the classical Poiseuille flow $U_{\max }$ located in the center of the channel is the appropriate outer velocity scale. An analysis of the present DNS data disclosed $C_{1}$ to be the bulk velocity $U_{B}(2.4)$ without an additional non-dimensional pre-factor.

In its final form the new logarithmic scaling law for the core region of the channel flow with wall transpiration is found to be

$$
\bar{U}_{1}=\frac{1}{\gamma} u_{\tau} \ln \left(\frac{x_{2}}{h}\right)+U_{B}
$$

or in deficit form

$$
\frac{\bar{U}_{1}-U_{B}}{u_{\tau}}=\frac{1}{\gamma} \ln \left(\frac{x_{2}}{h}\right)
$$

The new Lie symmetry induced scaling law (4.3) represents the velocity defect law that scales the data in the whole core region of the flow as may be taken from figure 9. This comparatively long log-region already appears at the low Reynolds numbers of $R e_{\tau}=250$ and becomes even longer as the Reynolds number is increased to $R e_{\tau}=850$ as it is obtained in figure $9(c, d)$. Most important, and as to be expected, the validity of (4.3) further increases with growing transpiration rate $v_{0}$, as it is shown in figure $9(a, b)$, until the latter becomes only an order of magnitude smaller than the streamwise velocity.

An indicator function shown on figure $9(e, f)$ confirms once again that the new mean velocity scaling law (4.3) is valid in the whole core region of the flow. In particular, it is found that the indicator function has a plateau in the core region, and the slope constant for the new core region log-law was found to be $\gamma=0.3$, as it is highlighted with a dashed line on figure $9(e, f)$. Further, the indicator function plot supports the fact that the scaling region of the new mean velocity scaling law increases with Reynolds numbers and moderate (up to $v_{0}^{+} \leqslant 0.16$ ) transpiration numbers. 
(a)
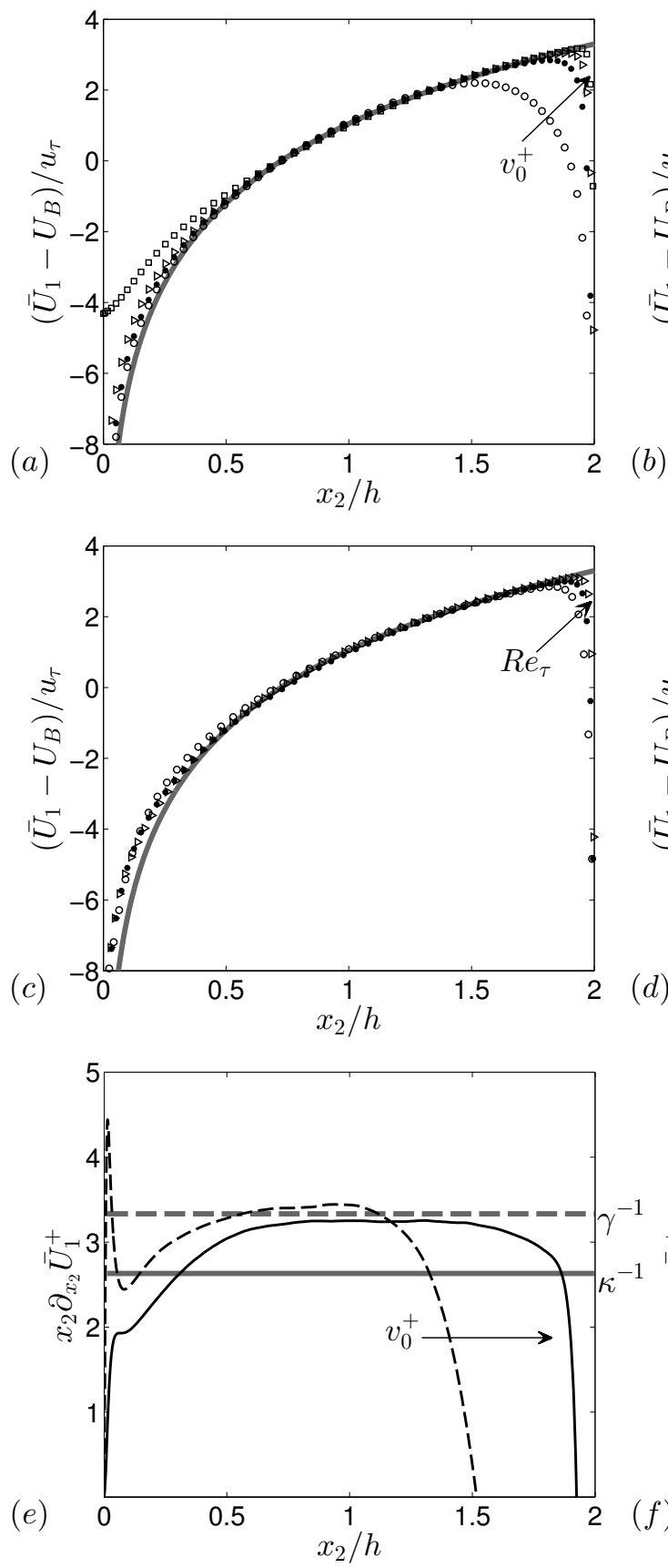
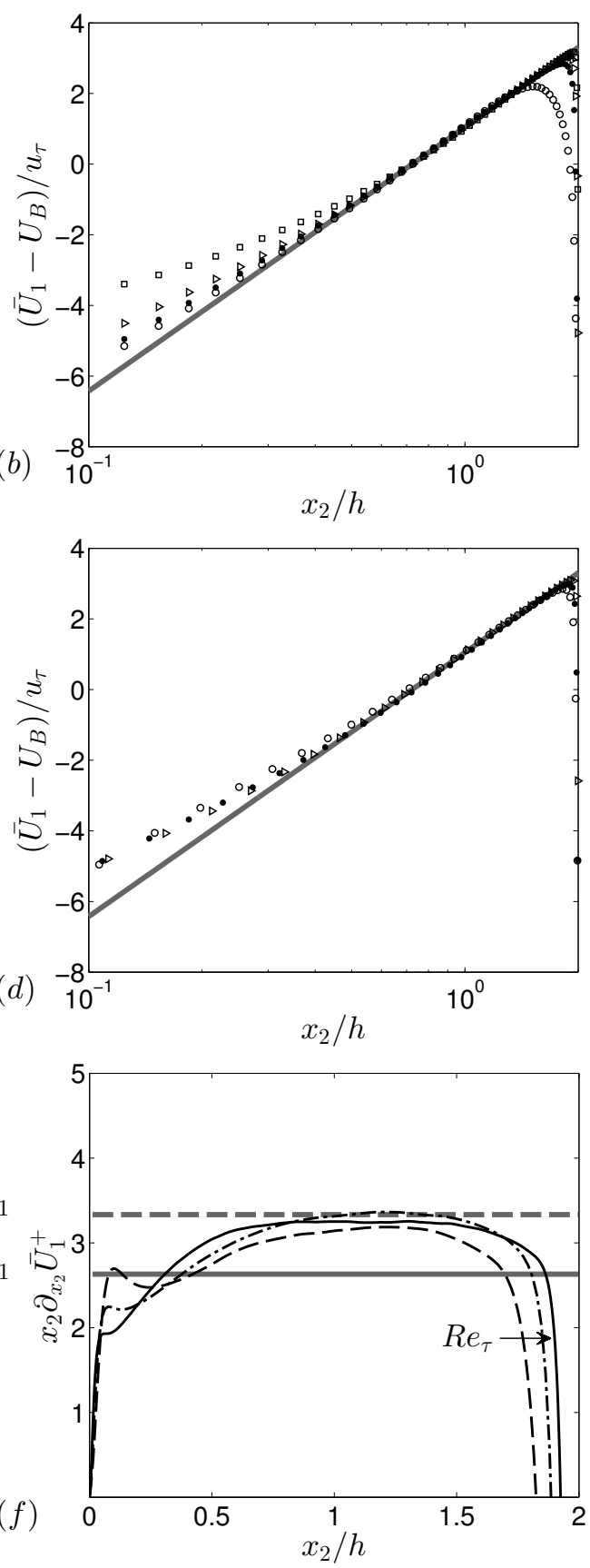

FiguRE 9. Mean velocity profiles at $(a, b)$ constant Reynolds number $R e_{\tau}=480$ : o, $v_{0}^{+}=0.05$; $\bullet, v_{0}^{+}=0.1 ; \triangleright, v_{0}^{+}=0.16, \square, v_{0}^{+}=0.26$; and $(c, d)$ constant transpiration rate $v_{0}^{+}=0.16$ : o, $R e_{\tau}=250 ; \bullet, R e_{\tau}=480 ; \triangleright, R e_{\tau}=850$. $(e, f)$ Indicator function $x_{2} \partial_{x_{2}} \bar{U}_{1}^{+}$. Grey lines represent slope constants used in the paper. For near-wall region: $\square, \kappa=0.38$; for core region: - = $\gamma=0.3$. (e) Constant Reynolds number $R e_{\tau}=850$ : in direction of an arrow: $\quad-\quad, v_{0}^{+}=0.05$; ,$- v_{0}^{+}=0.16$. $(f)$ Constant transpiration rate $v_{0}^{+}=0.16$ : in direction of an arrow: - - , $R e_{\tau}=250 ;-\cdot R e_{\tau}=480 ;-R e_{\tau}=850$. 


\section{Discussion and conclusions}

In the present paper we combined Lie symmetry analysis of the TPC equations and DNS to investigate the statistical characteristics of the turbulent channel flow with wall transpiration. Lie symmetry analysis revealed a new mean velocity logarithmic type of scaling law that, afterwards, has been confirmed in the center of the channel and studied in detail by DNS. For the derivation of the new log-law we used symmetry transformations which have been previously derived in the two- and MPC equations (Oberlack 2001; Oberlack \& Rosteck 2010). Although the completeness of the set of symmetry groups for turbulence statistics for the MPC equations has not been proven yet, it was sufficient to derive the new scaling law for the core region.

By using the new results from the DNS data it was found, that the slope constant $(\gamma)$ of the new log-law is different from the von Kármán constant and that its value is $\gamma=0.3$. Presence of the transpiration makes the log-region much longer than that of the velocity defect law for the classical channel flow. The new scaling law covers from $65 \%$ to $80 \%$ of the channel height depending on the transpiration rate. We observed an increase of the new log-law scaling region with increasing transpiration rate, though no first principles theory may be given for this behaviour. The theory does not provide any estimates of the location of the log-law. It might be possible to give an estimate for the validity such as the classical one for near-wall log, but we did not observe any clear behaviour and hence decided to avoid unvalidated speculations.

We found weak indications that the classical near-wall scaling laws, i.e. the linear law in the viscous sublayer and logarithmic law of the wall, may still exist on the blowing side though in slightly modified form. Further, we have found that at the suction wall the flow tends to relaminarize and at very high transpiration rates it becomes similar to the asymptotic suction velocity profile in exponential form, as it may be taken from figure $6(a, b)$. That indicates that the permeability of the channel walls strongly affects and, in fact, dominates the near-wall region and in particular the wall shear stress. This conclusion in a certain sense is not new, however, results obtained in the present paper show that at very high transpiration rates the properties of the near-wall region are completely changed, that can be taken from the figures $2(f), 3(f)$ and figure $6(a, b)$. We showed that the convective momentum transport $v_{0} \bar{U}_{1}$ exceeds both viscous and Reynolds stresses at the blowing wall and at the same time leads to an almost zero Reynolds stress at the suction wall. From the same figures and also from the fact that the new scaling law (4.3) was successfully validated with DNS data for moderate transpiration rates we may assume that the strong transpiration has also influence on the core region of the flow.

The importance of the present contribution may be contemplated in a somewhat larger context as another brick in the turbulence theory based on Lie symmetries applied to the multi-point correlation equation the reason being twofold. First, before the entire project started and before any DNS was conducted the new log-scaling laws was forecasted from pure theoretical grounds. Second, the basis for the new log-law is partially based on two of the new statistical groups which have no direct counterpart in the Euler and NavierStokes equations for the instantaneous velocities and hence have yet been proven one more time to be crucial for our general understanding of the statistics of turbulence.

This work was supported by the DFG under the grant number KH 257/2-1 (2010). The computations were performed on the HHLR IBM Regatta supercomputer at TU Darmstadt, on the FUCHS cluster at the University of Frankfurt-am-Main and on the SuperMUC Petascale System at Leibniz Supercomputing Centre (LRZ). Special thanks are due to M. Rampp for providing us an account on the HP Visualization Cluster at the 
Rechenzentrum Garching (RZG) of the Max Planck Institute for Plasmaphysics (IPP) were we performed visualizations of the data. Finally, the authors would like to thank Y. Wang, M. Frewer and A. Rosteck for their valuable comments.

\section{REFERENCES}

Del Álamo, J. C. \& JimÉnez, J. 2003 Spectra of the very large anisotropic scales in turbulent channels. Phys of Fluids 15 (6), 41-44.

del Álamo, J. C., Jiménez, J., Zandonade, P. \& Moser, R. D. 2004 Scaling of the energy spectra of turbulent channels. J. Fluid Mech. 500, 135-144.

Antonia, R. S., Krishnamoorthy, L. V., Fulachier, L., Anselmet, F. \& Benabid, T. 1986 Influence of wall suction on coherent structures in a turbulent boundary layer. pp. 346-349. 9th Australasian Fluid Mechanics Conference, Auckland, Australia.

Avsarkisov, V. S., Oberlack, M. \& Khujadze, G. 2011 Turbulent poiseuille flow with wall transpiration: Analytical study and direct numerical simulation. J. of Phys.: Conf. Ser. 318 (022004), 1-6.

Black, T. J. \& SARneCKI, A. J. 1958 The turbulent boundary layer with suction or injection. Tech. Rep. 20. Cambrige University, Engineering Department.

Bluman, G. W., Cheviakov, A. F. \& Anco, S. C. 2010 Application of Symmetry Methods to Partial Differential Equations. Springer.

Chung, Y. M. \& Sung, H. J. 2001 Initial relaxation of spatially evolving turbulent channel flow with blowing and suction. AIAA Journal 39 (11), 2091-2099.

Chung, Y. M., Sung, H. J. \& Krogstad, P.-A. 2002 Modulation of near-wall turbulence structure with wall blowing and suction. AIAA Journal 40 (8), 1529-1535.

Drazin, P. G. \& Riley, N. 2006 The Navier-Stokes equations: a classification of flows and exact solutions. Cambridge University Press.

Fife, P., Klewicki, J. C. \& WeI, T. 2009 Time averaging in turbulence settings may reveal an infinite hierarchy of length scales. J. Disc Cont. Dyn Sys. 24 (3), 781-807.

Griffith, A. A. \& Meredith, F. W. 1936 Possible improvement in aircraft performance due to use of boundary layer suction. Tech. Rep. 2315. Aero. Res. Counc., London.

Hanjalić, K. \& Launder, B. E. $1972 a$ Fully developed asymmetric flow in a plane channel. J. Fluid Mech. 51(2), 301-335.

Hanjalić, K. \& Launder, B. E. $1972 b$ Reynolds-stress model of turbulence and its application to thin shear flows. J. Fluid Mech. 52(4), 609-638.

Hinze, J. O. 1959 Turbulence, An Introduction to Its Mechanism and Theory. McGraw-Hill.

Hoyas, S. \& Jiménez, J. 2006 Scaling of the velocity fluctuations in turbulent channels up to $R e_{\tau}=2003$. Phys. Fluids 18, 011702-1-4.

JACKSON, P.S. 1981 On the displacement height in the logarithmic velocity profile. J. Fluid Mech. 111, 15-25.

Jiménez, J., Uhlmann, M., Pinelli, M. \& Kawahara, G. 2001 Turbulent shear flow over active and passive porous surfaces. J. Fluid Mech. 442, 89-117.

Johnstone, R., Coleman, G. N. \& Spalart, P. R. 2010 The resilience of the logarithmic law to pressure gradients: evidence from direct numerical simulation. J. Fluid Mech.643, $163-175$.

Kametani, Y. \& Fukagata, K. 2011 Direct numerical simulation of spatially developing turbulent boundary layer with uniform blowing or suction. J. Fluid Mech. 681, 154-172.

von KÁrmán, TH. 1930 Mechanische Ähnlichkeit und Turbulentz. Nachr. Ges. Wiss. Göettingen, Math-Phys. Kl. 68.

von Kármán, Th. \& Howarth, L. 1938 On the statistical theory of isotropic turbulence. Proc. R. Soc. Lond. A. 164, 192-215.

Keller, L. \& Friedmann, A. 1924 Differentialgleichungen für die turbulente Bewegung einer kompressiblen Flüssigkeit. Proc. First. Int. Congr. Appl. Mech. pp. 395-405.

Kim, J., Moin, P. \& Moser, R. 1987 Turbulence statistics in fully developed channel flow at low Reynolds number. J. Fluid Mech. 177, 133-166.

KLewicki, J. C. 2013 Self-similar mean dynamics in turbulent wall flows. J. Fluid Mech. 718, 596-612. 
Kraichnan, R. H. 1965 Lagrangian-history closure approximaiton for turbulence. Phys. Fluids $8(4), 575-598$.

Launder, B. E., Reece, G. J. \& Rodi, W. 1975 Progress in the development of a Reynoldsstress turbulence closure. J. Fluid Mech. 68(3), 537-566.

LELE, S. K. 1992 Compact finite difference schemes with spectral-like resolution. J. of Comp. Phys. 103, 16-42.

Lindgren, B., Österlund, J. M. \& Johansson, A. V. 2004 Evaluation of scaling laws derived from lie group symmetry methods in zero-pressure-gradient turbulent boundary layers. J. Fluid Mech. 502, 127-152.

MсСомв, W. D. 1990 The Physics of Fluid Turbulence. Oxford University Press.

Mickley, H. S. \& Davis, R.S. 1957 Momentum transfer for flow over a flat plate with blowing. Tech. Rep. 4017. MIT.

Millikan, C. B. 1939 A critical discussion of turbulent flows in channels and circular tubes. Proc. Vth Int. Congr. Appl. Mech. pp. 386-392.

Nakabayashi, K., Кiтон, O. \& Katoh, Y. 2004 Similarity laws of velocity profiles and turbulence characteristics of couette-poiseuille turbulent flows. J. Fluid Mech. 507, 43-69.

Nikitin, N.V. \& PAVEL'Ev, A.A. 1998 Turbulent flow in a channel with permeable walls. DNS and results of three-parameter-model. J. Fluid Mech. 33 (6), 826-832.

OBerlack, M. 2000 Symmetrie, Invarianz und Selbstähnlichkeit in der Turbulenz. Habilitation thesis .

OBerlack, M. 2001 A unified approach for symmetries in plane parallel turbulent shear flows. J. Fluid Mech. 427, 299-328.

Oberlack, M. \& Rosteck, A. 2010 New statistical symmetries of the multi-point equations and its importance for turbulent scaling laws. Dis. and Cont. Dyn. Sys. S 3 (3).

Rosteck, A. \& Oberlack, M. 2011 Lie algebra of the symmetries of the multi-point equations in statistical turbulence theory. J. Nonlinear Math. Phys. 18, 251-264.

Schlatter, P. \& ÖRL Ü, R. 2011 Turbulent asymptotic suction boundary layers studied by simulation. J. Phys. Conference Series 318, 1-10.

Stevenson, T. N. 1963a A law of the wall for turbulent boundary layers with suction or injection. Tech. Rep. 166. The College of Aeronautics Cranfield.

Stevenson, T. N. $1963 b$ A modified velocity defect law for turbulent boundary layers with injection. Tech. Rep. 170. The College of Aeronautics Cranfield.

Sumitani, Y. \& Kasagi, N. 1995 Direct numerical simulation of turbulent transport with uniform wall injection and suction. AIAA Journal 33 (7), 1220-1228.

Telbany, M. M. M. El \& Reynolds, A. J. 1981 Turbulence in plane channel flows. J. Fluid Mech. 111, 283-318.

Tennekes, H. 1965 Similarity laws for turbulent boundary layers with suction or injection. J. Fluid Mech. 21(4), 689-703.

Tennekes, H. \& Lumley, J. L. 1972 A First Course in Turbulence. The MIT Press.

Vigdorovich, I. \& Oberlack, M. 2008 Analytical study of turbulent Poiseuille flow with wall transpiration. Phys. Fluids 20, 055102-1-9.

Wei, T., Fife, P. \& KLEWiCKI, J. 2007 On scaling the mean momentum balance and its solutions in turbulent couette-poiseuille flow. J. Fluid Mech. 573, 371-398.

Wosnik, M., Castillo, L. \& George, W. K. 2000 A theory for turbulent pipe and channel flows. J. Fluid Mech. 421, 115-145.

Zhapbasbayev, U. \& Isakhanova, G. 1998 Developed turbulent flow in a plane channel with simultaneous injection through one porous wall and suction through the other. J. Appl. Mech. Tech. Phys. 39, 53-59. 